\title{
Design of shift-invariant nonuniform cosine-modulated filter bank with arbitrary integer sampling factors
}

\author{
Lili Liang*, Han Liu and Fengping Wang
}

\begin{abstract}
Shift-invariance and unequal frequency partition are two desirable properties in many signal/image processing applications. In this paper, we treat a simple but efficient approach to design shift-invariant nonuniform cosine-modulated filter bank (SI-NCMFB) with arbitrary integer sampling factors. It consists of two subsystems where the first one is obtained by merging the consecutive subbands of a cosine-modulated filter bank, and the second one is derived under the condition of shift-invariance. By imposing the significant aliasing in the two subsystems to cancel each other structurally, the proposed SI-NCMFB achieves the properties of shift-invariance and flexible frequency partition. Since both the first and second subsystems come from just one prototype filter, the SI-NCMFB design is reduced to a filter design, leading to low design complexity. Furthermore, we extend the SINCMFB to two dimensions by separable operations. Unlike traditional separable transforms, the resulting 2D subbands have flexible directional-selectivity which is highly expected in image representation. Several simulation experiments are given to verify the proposed SI-NCMFB and the designed SI-NCMFB is of near-perfect reconstruction.
\end{abstract}

\section{Key words}

Arbitrary integer sampling factor, directional-selectivity, flexible frequency partition, shiftinvariance

\section{Introduction}

Manuscript received June 1, 2015. This work was supported in part by the National Science Foundation of China under Grant 61403305 and Grant 61174101, in part by the Doctoral Program Foundation of the Ministry of Education of China under Grant 20126118110004 and Grant 20136118120005, in part by the Scientific Research Program Funded by Shaanxi Provincial Education Department under Grant 14JK1543, and in part by the Doctor Research Start Fund of Xi'an University of Technology under Grant 105-211307.

Lili Liang (Corresponding author), Han Liu and Fengping Wang are with the Faculty of Automation and Information Engineering, Xi'an University of Technology, Xi'an 710048, China (e-mail: 1lliang@xaut.edu.cn, liuhan@xaut.edu.cn, fpwang@stu.xaut.edu.cn; phone: 86-29-82312431; fax: 86-29-82312431). 
Nonuniform filter banks (NUFBs) as shown in Fig. 1 find wide application in the fields of signal/image processing due to their unequal frequency partition [1-4]. Over several decades, significant efforts have been devoted to the critically sampled NUFB and some efficient design methods including the combination and stretching are proposed [5-11]. The critically sampled NUFBs are successfully used in subband coding since their output size is equal to the input size [12]. However, the critical sampling causes significant inband aliasing which leads to shift-variance at each subband output [5], that is, a small time-shift in the input signal will cause major variations in the subband outputs. Moreover, the sampling factors have to form a compatible or feasible set to cancel the aliasing completely or approximately, which makes some frequency partition schemes unrealizable [6]. These limitations prevent them from being used in many applications where the properties of shift-invariance and flexible frequency partition are required, such as noise reduction, echo cancellation, and feature extraction.

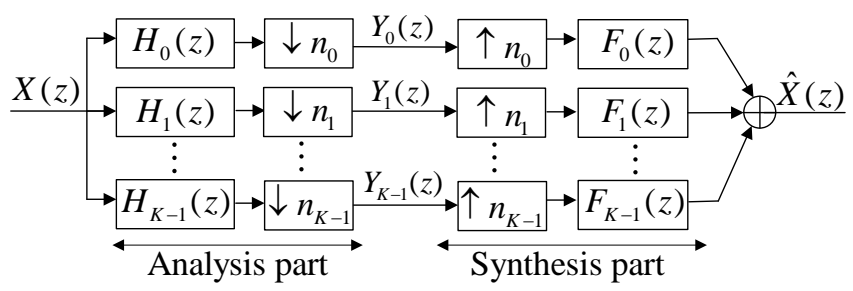

Fig. 1. Structure of a $K$-channel traditional NUFB. On the analysis part, the input signal $X(z)$ is filtered by the analysis filters $H_{k}(z)$ and downsampled by $n_{k}$ to produce the $K$ unequal frequency subbands $Y_{k}(z)$. On the synthesis part, the $K$ subbands are recombined to form the output signal $\hat{X}(z)$ by using the upsampling factors $n_{k}$ and the synthesis filters $F_{k}(z), k=0,1, \cdots, K-1$.

Due to the fact that oversampling can reduce significant inband aliasing and alleviate the shiftvariance problem, some researchers relax the critical sampling to design perfect-reconstruction (PR) or near-PR (NPR) oversampled NUFBs. Since the PR is overly restrictive in some practical applications and the PR condition is often in conflict with filter stopband attenuation [13], the oversampled NPR NUFBs have received much more attention and become the main design target. The existing design methods for oversampled NPR NUFBs can be broadly classified into three categories: joining [14-16], frequency warping [17-19], and merging [20,21]. The first category obtains an oversampled NPR NUFB by firstly designing several uniform filter banks (UFBs) and then a set of transition filters to join the consecutive sections of these UFBs [14-16]. To perform a desired 
nonuniform frequency partition, an individual UFB for each subband that has a different bandwidth from the others and the corresponding transition filter for each pair of UFBs have to be designed. This will lead to somewhat high design complexity, especially when the frequency partition is highly nonuniform. The second category designs an oversampled NPR NUFB by replacing all the delay elements of a uniform DFT filter bank with all-pass filters $[17,18]$. The all-pass filters warp the uniform frequency partition to a nonuniform form with the order controlling the partition flexibility. The higher the order of the all-pass filters, the more flexible the frequency partition of the oversampled NUFB is. However, the high order means high implementation complexity and long system delay. In [19], Parfieniuk and Petrovsky combined frequency warping and subband merging to offer flexible frequency partition while minimizing the implementation complexity, but at the expense of loss in partition accuracy. The third category obtains an oversampled NUFB by firstly designing a UFB and then directly merging an appropriate number of consecutive subbands of this UFB [20,21]. It has low design and implementation complexity for involving one UFB and a direct merging operation. To remove the significant inband aliasing and make the designed NUFBs shift-invariant, all those three category methods restrict that there is no passband or transition band overlap between the synthesis filter and the shifted analysis filters in each channel. However, this non-overlap realization requires the frequency partition to be feasible $[18,19]$. As for the NUFB with nonfeasible frequency partition, those three category methods become invalid since the significant inband aliasing cannot be mitigated theoretically, which limits the flexibility of the frequency partition.

Motivated by the ideas of achieving nonuniform frequency partition in [7] and eliminating significant inband aliasing in [22], we present a simple but efficient design method for an alternative oversampled NPR NUFB. It has the shift-invariance property and arbitrary integer sampling factors at the cost of limited redundancy. Different from the traditional NUFB as shown in Fig. 1, the proposed shift-invariant nonuniform cosine-modulated filter bank (SI-NCMFB) is composed of two subsystems. The first subsystem is designed by directly merging the consecutive subbands of a cosine-modulated filter bank (CMFB) and the second one is derived under the condition of shift-invariance. By selecting the modulation technique properly, the significant aliasing in each channel of the first subsystem can be cancelled structurally by that in the corresponding channel of the second subsystem. As a result, 
the shift-invariance property is achieved, and meanwhile the integer sampling factors can be arbitrary. The arbitrary integer sampling factors mean that both the feasible and nonfeasible frequency partitions can be performed. Since both the first and second subsystems come from just one prototype filter, the proposed SI-NCMFB design is simplified into a prototype design, reducing the design complexity significantly. Furthermore, we extend the SI-NCMFB from one-dimension to two-dimensions via separable operations. The resulting 2D subbands have flexible directional-selectivity, which is highly desired in image representation.

The remainder of the paper is organized as follows. Section 2 introduces the structure of SINCMFB and the condition of shift-invariance. Section 3 considers the design of the two subsystems as well as the reconstruction and implementation of the whole system. Section 4 analyzes the directional-selectivity of SI-NCMFB in two-dimensions. Section 5 presents several simulation experiments to demonstrate the validity of SI-NCMFB, and some conclusions are given in Section 6. Note that in our work, all the involved filters are assumed to be FIR.

\section{Construction of SI-NCMFB}

In this section, we first consider the structure of SI-NCMFB with arbitrary integer sampling factors and then give the condition for it to have shift-invariance property.

\subsection{Structure of SI-NCMFB}

In [22], the dual-tree complex wavelet transform (DTCWT) is constructed with two parallel trees. It achieves the shift-invariance by imposing the aliasing in both trees to cancel each other approximately (it will involve IIR filters for the exact aliasing cancellation which is beyond the scope of our work). Motivated by the idea of DTCWT, we construct a $K$-channel SI-NCMFB with two subsystems and make it shift-invariant in the same way as DTCWT. Fig. 2 illustrates this proposed SI-NCMFB with $H_{k}(z)$ and $F_{k}(z)$ denoting the analysis and synthesis filters of the first subsystem, and $H_{k}^{\prime}(z)$ and $F_{k}^{\prime}(z)$ representing the filters of the second subsystem. The positive integers $n_{k}, k=0,1, \cdots, K-1$, are integer sampling factors and satisfy $\sum_{k=0}^{K-1} 1 / n_{k}=1$. It is obvious that the SI-NCMFB is an oversampled system and has the redundancy of 2 due to the two subsystems. According to the 
sampling factors, the frequency support of $H_{k}(z)$ and $H_{k}^{\prime}(z)$ is set to be $\left[-\pi / n_{0}, \pi / n_{0}\right]$ for $k=0$ and $\left[-\sum_{i=0}^{k} \pi / n_{i},-\sum_{i=0}^{k-1} \pi / n_{i}\right] \cup\left[\sum_{i=0}^{k-1} \pi / n_{i}, \sum_{i=0}^{k} \pi / n_{i}\right]$ for $k=1,2, \ldots, K-1$, as sketched in Fig. 3. As for the synthesis filters $F_{k}(z)$ and $F_{k}^{\prime}(z)$, they are set to have the similar magnitude responses with their corresponding analysis filters. Here it should be noted that, in our work, the frequency support represents the frequency interval between the lower and upper cut-off frequencies.

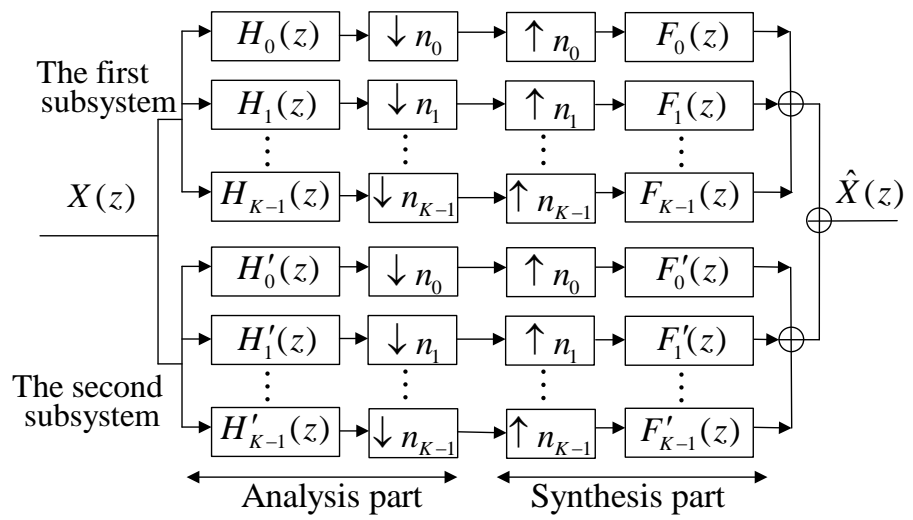

Fig. 2. Structure of the proposed SI-NCMFB.

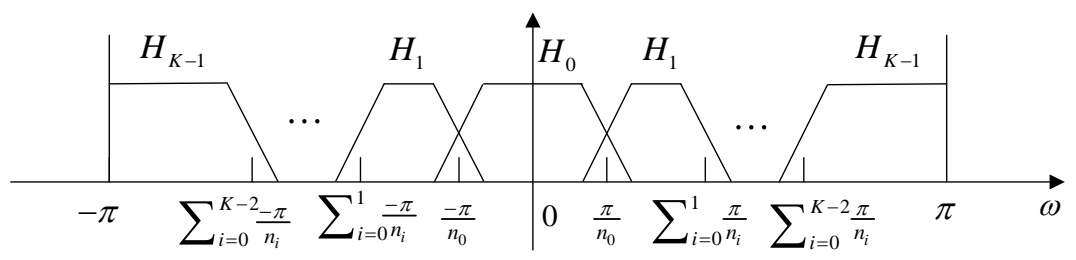

Fig. 3. Magnitude responses of the analysis filters $H_{k}(z)$ and $H_{k}^{\prime}(z)$.

As mentioned in Introduction, the prerequisite for the traditional oversampled NUFB to achieve shift-invariance is the feasible frequency partition. Here we give a brief explanation for it. Considering the $k$ th channel of Fig. 1, the downsampling stretches $H_{k}(z)$ by a factor of $n_{k}$ and creates $n_{k}-1$ copies of this stretched $H_{k}(z)$ by shifting it uniformly in successive amounts of $2 \pi$. To achieve shift-invariance, the stretched $H_{k}(z)$ and its shifted versions should have no significant overlap. This requires the frequency support of $H_{k}(z)$ to be in the region of $\left[-r_{k} \pi / n_{k},-\left(r_{k}-1\right) \pi / n_{k}\right] \cup\left[\left(r_{k}-1\right) \pi / n_{k}, r_{k} \pi / n_{k}\right]$ as shown in Fig. 4, where $r_{k}$ is a position integer $[19,20]$. Otherwise, they will overlap in the passband and lead to significant aliasing that cannot be eliminated in theory. However, for the proposed SI-NCMFB, by imposing the aliasing in the first and second subsystems to cancel each other approximately, the shift-invariance will always be achieved 
no matter whether the frequency partition is feasible or not.

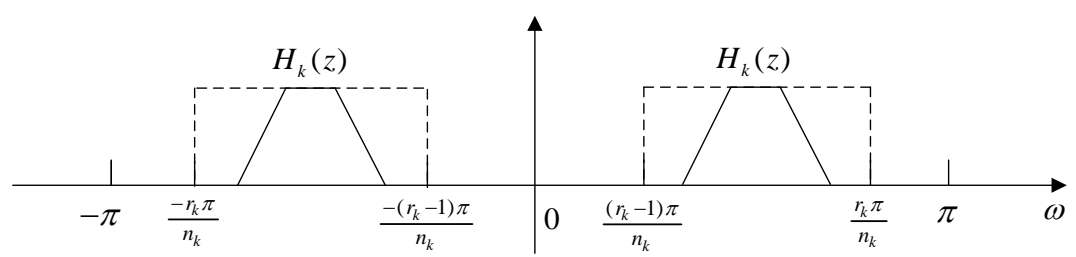

Fig. 4. Illustration of feasible frequency partition with the dashed line representing a guard band. When downsampled by $n_{k}$, the stretched $H_{k}(z)$ will have no passband overlap with its shifted versions if the frequency support of $H_{k}(z)$ is in the guard band region.

\subsection{Shift-invariance condition}

Without loss of generality, we analyze the $k$ th channels of the first and second subsystems as shown in Fig. 5. For convenient analysis, its shift-invariance is discussed in the $Z$ domain and the inputoutput relationship is written as

$$
\hat{X}_{k}(z)=A_{k, 0}(z) X(z)+\sum_{l=1}^{n_{k}-1} A_{k, l}(z) X\left(z W_{n_{k}}^{l}\right),
$$

where $W_{n_{k}}=e^{-j 2 \pi / n_{k}}$ and

$$
A_{k, l}(z)=\frac{1}{n_{k}}\left[H_{k}\left(z W_{n_{k}}^{l}\right) F_{k}(z)+H_{k}^{\prime}\left(z W_{n_{k}}^{l}\right) F_{k}^{\prime}(z)\right]
$$

The terms $A_{k, l}(z)$ with $X\left(z W_{n_{k}}^{l}\right)$ for $l>0$ represent aliasing which is caused by downsampling. Such a system is shift-invariant if an integer time-shift $m$ in the input signal causes only the same time-shift in the output, or equivalently in the $Z$ domain if the output in response to $z^{-m} X(z)$ is $z^{-m} \hat{X}_{k}(z)$. Hence, in order for (1) to be shift-invariant, those aliasing terms have to be negligible.

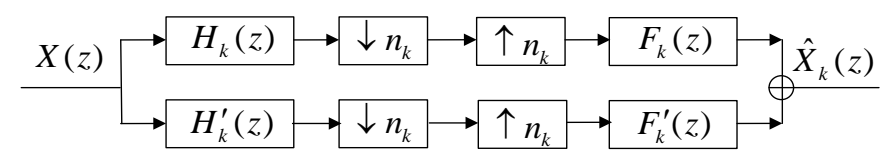

Fig. 5. The $k$ th channels of the two subsystems.

Observing (2), it can be seen that if $H_{k}\left(z W_{n_{k}}^{l}\right) F_{k}(z)$ and $H_{k}^{\prime}\left(z W_{n_{k}}^{l}\right) F_{k}^{\prime}(z)$ have the same magnitude responses and their phase responses differ by $\pi$, they can cancel each other and the aliasing terms $A_{k, l}(z)$ can thereby be eliminated. In [22], Kingsbury discussed this issue thoroughly and pointed out that it can be performed by restricting the impulse responses of $H_{k}^{\prime}(z)$ and $F_{k}^{\prime}(z)$ to be the Hilbert 
transforms of the impulse responses of $H_{k}(z)$ and $F_{k}(z)$, respectively. Based on the discussion, we set that

$$
\begin{aligned}
& H_{k}(z)=P_{k}(z)+P_{k}^{*}(z), \\
& H_{k}^{\prime}(z)=-j\left[P_{k}(z)-P_{k}^{*}(z)\right], \\
& F_{k}(z)=Q_{k}(z)+Q_{k}^{*}(z), \\
& F_{k}^{\prime}(z)=j\left[Q_{k}(z)-Q_{k}^{*}(z)\right],
\end{aligned}
$$

where the superscript $*$ denotes coefficient conjugation. $P_{k}(z)$ and $Q_{k}(z)$ are set to correspond to the positive frequency passbands of $H_{k}(z)$ and $F_{k}(z)$ and thus are two complex filters with the positive frequency support $\left[\sum_{i=0}^{k-1} \pi / n_{i}, \sum_{i=0}^{k} \pi / n_{i}\right]$. Accordingly, the conjugations $P_{k}^{*}(z)$ and $Q_{k}^{*}(z)$ have the negative support $\left[-\sum_{i=0}^{k} \pi / n_{i},-\sum_{i=0}^{k-1} \pi / n_{i}\right]$ and are related to the negative frequency passbands of $H_{k}(z)$ and $F_{k}(z)$. Substituting (3) into (1), we have

$$
\hat{X}_{k}(z)=\frac{2}{n_{k}} \sum_{l=0}^{n_{k}-1}\left[P_{k}\left(z W_{n_{k}}^{l}\right) Q_{k}(z)+P_{k}^{*}\left(z W_{n_{k}}^{l}\right) Q_{k}^{*}(z)\right] X\left(z W_{n_{k}}^{l}\right) .
$$

$P_{k}\left(z W_{n_{k}}^{l}\right)$ is a shifted version of $P_{k}(z)$ with the frequency offset $2 \pi l / n_{k}$ and thus has the support $\left[2 \pi l / n_{k}+\sum_{i=0}^{k-1} \pi / n_{i}, 2 \pi l / n_{k}+\sum_{i=0}^{k} \pi / n_{i}\right]$. Fig. 6 sketches the frequency supports of $P_{k}\left(z W_{n_{k}}^{l}\right)$ and $Q_{k}(z)$. It is observed that since $P_{k}\left(z W_{n_{k}}^{l}\right)$ and $Q_{k}(z)$ have the bandwidth $\pi / n_{k}$, the passbands and transition bands of $P_{k}\left(z W_{n_{k}}^{l}\right)$ overlap only with the stopband of $Q_{k}(z)$ if the passbands and transition bands of $P_{k}(z)$ and $Q_{k}(z)$ lie within the frequency interval [ $\left.\sum_{i=0}^{k-1} \pi / n_{i}-0.5 \pi / n_{k}, \sum_{i=0}^{k} \pi / n_{i}+0.5 \pi / n_{k}\right]$. In this case, $P_{k}\left(z W_{n_{k}}^{l}\right) Q_{k}(z)$, although not equal to zero, can be made very small by attenuating the stopband responses of $P_{k}(z)$ and $Q_{k}(z)$. As a result, all the aliasing terms $A_{k, l}(z)$ can be negligible and the expression (4) can be simplified as

$$
\hat{X}_{k}(z)=\frac{2}{n_{k}}\left[P_{k}(z) Q_{k}(z)+P_{k}^{*}(z) Q_{k}^{*}(z)\right] X(z),
$$

which is obviously shift-invariant.

Further, it should be noted that during the above analysis, the frequency supports of $H_{k}(z)$ and $F_{k}(z)$ are not restricted in the interval $\left[\left(r_{k}-1\right) \pi / n_{k}, r_{k} \pi / n_{k}\right]$ as shown in Fig. 4. It means that no 
matter whether the frequency partition is feasible or not, the aliasing terms can be made negligible as long as the two subsystems satisfy (3). In other words, the proposed SI-NCMFB can have shiftinvariance property and arbitrary integer sampling factors under the condition of (3).

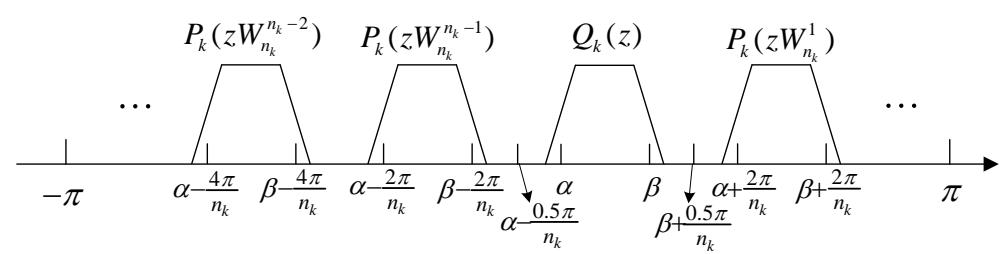

Fig. 6. Magnitude responses of $P_{k}\left(z W_{n_{k}}^{l}\right)$ and $Q_{k}(z)$, where $\alpha=\sum_{i=0}^{k-1} \pi / n_{i}$ and $\beta=\sum_{i=0}^{k} \pi / n_{i}$.

\section{The two subsystems}

\subsection{Design of the first and second subsystems}

Merging outputs of a UFB is a simple but efficient method for NUFB design [7]. It performs a nonuniform frequency partition by firstly designing a uniform CMFB and then merging its consecutive subbands. In our work, we employ the merging method to design the first subsystem and then build the second subsystem to satisfy (3).

Let $M$ be the least common multiple (lcm) of the integer sampling factors $\left\{n_{0}, n_{1}, \cdots, n_{K-1}\right\}$ and $m_{k}=M / n_{k}$. To construct the first subsystem, we first design an $M$-channel uniform CMFB with the analysis filters $H_{i}^{c}(z)$ and the synthesis filters $F_{i}^{c}(z)$ as shown in (6), [23]

$$
\begin{aligned}
& H_{i}^{c}(z)=a_{i} U_{i}(z)+a_{i}^{*} U_{i}^{*}(z), \\
& F_{i}^{c}(z)=a_{i}^{*} U_{i}(z)+a_{i} U_{i}^{*}(z),
\end{aligned}
$$

where $a_{i}=e^{j(-1)^{i} / 4}$ and $U_{i}(z)$ is defined as

$$
U_{i}(z)=W_{2 M}^{(i+0.5) N / 2} P\left(z W_{2 M}^{i+0.5}\right)
$$

$P(z)$ is a linear-phase prototype filter with the frequency support $[-\pi / 2 M, \pi / 2 M]$ and the order $N$. $U_{i}(z)$ represents a shifted version of $P(z)$ with the frequency offset $\pi(i+0.5) / M$ and thus has the frequency support $[i \pi / M,(i+1) \pi / M]$. The frequency support of $H_{i}^{c}(z)$ and $F_{i}^{c}(z)$ is accordingly $[-(i+1) \pi / M,-i \pi / M] \cup[i \pi / M,(i+1) \pi / M]$. All the analysis and synthesis filters are modulated versions of $P(z)$. 
Subsequently, we merge the $m_{k}$ consecutive subbands of the pre-designed CMFB and obtain the $k$ th subband of the first subsystem below,

$$
\begin{aligned}
& H_{k}(z)=\frac{1}{\sqrt{m_{k}}} \sum_{i=l_{k}}^{l_{k}+m_{k}-1}\left(a_{i} U_{i}(z)+a_{i}^{*} U_{i}^{*}(z)\right), \\
& F_{k}(z)=\frac{1}{\sqrt{m_{k}}} \sum_{i=l_{k}}^{l_{k}+m_{k}-1}\left(a_{i}^{*} U_{i}(z)+a_{i} U_{i}^{*}(z)\right),
\end{aligned}
$$

where $l_{k}=0$ for $k=0$ and $l_{k}=\sum_{i=0}^{k-1} m_{i}$ for $k=1,2, \cdots, K-1$. According to (8) and the frequency support of $U_{i}(z)$, we derive that $\sum_{i=l_{k}}^{l_{k}+m_{k}-1} a_{i} U_{i}(z)$ and $\sum_{i=l_{k}}^{l_{k}+m_{k}-1} a_{i}^{*} U_{i}(z)$ have the positive frequency support $\left[\sum_{i=0}^{k-1} \pi / n_{i}, \sum_{i=0}^{k} \pi / n_{i}\right]$, while $\sum_{i=l_{k}}^{l_{k}+m_{k}-1} a_{i} U_{i}^{*}(z)$ and $\sum_{i=l_{k}}^{l_{k}+m_{k}-1} a_{i}^{*} U_{i}^{*}(z)$ have the negative frequency support $\left[-\sum_{i=0}^{k} \pi / n_{i},-\sum_{i=0}^{k-1} \pi / n_{i}\right]$. This is in accordance with the frequency partition of the SI-NCMFB as sketched in Fig. 3.

Observing (3) and (8), it can be seen that $\sum_{i=l_{k}}^{l_{k}+m_{k}-1} a_{i} U_{i}(z)$ corresponds to $P_{k}(z)$ and $\sum_{i=l_{k}}^{l_{k}+m_{k}-1} a_{i}^{*} U_{i}(z)$ is related to $Q_{k}(z)$. Consequently, the analysis and synthesis filters of the second subsystem is defined as

$$
\begin{aligned}
& H_{k}^{\prime}(z)=-\frac{j}{\sqrt{m_{k}}} \sum_{i=l_{k}}^{l_{k}+m_{k}-1}\left(a_{i} U_{i}(z)-a_{i}^{*} U_{i}^{*}(z)\right), \\
& F_{k}^{\prime}(z)=\frac{j}{\sqrt{m_{k}}} \sum_{i=l_{k}}^{l_{k}+m_{k}-1}\left(a_{i} U_{i}(z)-a_{i}^{*} U_{i}^{*}(z)\right),
\end{aligned}
$$

which satisfy the shift-invariance condition (3). By now, both the two subsystems are derived and the resulting SI-NCMFB can have the shift-invariance property and arbitrary integer sampling factors. In addition, Eqs. (8) and (9) also imply that the first and second subsystems come from just one prototype filter, which will reduce the design complexity significantly.

\subsection{Reconstruction performance}

The reconstruction process performed in a filter bank generally suffers from aliasing error, amplitude distortion and phase distortion. For a NPR system, the three errors should be small enough to be ignored. In what follows, we consider the reconstruction performance of SI-NCMFB. 
Going back to Fig. 2, the input and output relationship of the SI-NCMFB is

$$
\begin{aligned}
\hat{X}(z) & =\sum_{k=0}^{K-1} A_{k, 0}(z) X(z)+\sum_{k=0}^{K-1} \sum_{l=1}^{n_{k}-1} A_{k, l}(z) X\left(z W_{n_{k}}^{l}\right) \\
& =T(z) X(z)+A(z),
\end{aligned}
$$

where $A_{k, l}(z)$ is given in (2). $T(z)$ is called the overall transfer function and $A(z)$ is the total aliasing. Since $A_{k, l}(z)$ can be negligible as discussed in Section 2.2, the total aliasing $A(z)$ can also be negligible.

Now we analyze the overall transfer function. Substituting (8) and (9) into (10), $T(z)$ has the following expression,

$$
T(z)=\frac{2}{M} \sum_{k=0}^{K-1} \sum_{i=l_{k}}^{l_{k}+m_{k}-1 l_{l}+m_{k}-1} \sum_{i^{\prime}=l_{k}}^{(}\left(a_{i} a_{i^{\prime}}^{*} U_{i}(z) U_{i^{\prime}}(z)+a_{i}^{*} a_{i^{\prime}} U_{i}^{*}(z) U_{i^{\prime}}^{*}(z)\right) .
$$

Assuming that $U_{i}(z)$ has high stopband attenuation and there is no passband or transition band overlap between $U_{i}(z)$ and $U_{i^{\prime}}(z)$ when $\left|i-i^{\prime}\right|>1, T(z)$ can be simplified into

$$
T(z) \approx \frac{2}{M} \sum_{i=0}^{M-1}\left(U_{i}^{2}(z)+U_{i}^{* 2}(z)\right)
$$

Since $P(z)$ is a linear-phase prototype filter and $U_{i}(z)=W_{2 M}^{(i+0.5) N / 2} P\left(z W_{2 M}^{i+0.5}\right)$, we derive that $U_{i}^{*}(z)=(-1)^{N} U_{2 M-1-i}(z)$ and $U_{i}(z)=z^{-N} U_{i}^{*}\left(z^{-1}\right)$ which is shown in Appendix A. Substituting them, $T(z)$ can be further rewritten as

$$
T(z) \approx z^{-N}|T(z)|,
$$

where

$$
|T(z)|=\frac{2}{M} \sum_{i=0}^{M-1}\left(\left|U_{i}(z)\right|^{2}+\left|U_{i}^{*}(z)\right|^{2}\right)=\frac{2}{M} \sum_{i=0}^{2 M-1}\left|U_{i}(z)\right|^{2}
$$

$T(z)$ is obviously of linear-phase and the proposed SI-NCMFB is free from phase distortion.

As for the amplitude distortion, it depends on the flatness of $|T(z)|$. That is to say, $|T(z)|$ should be as close to 1 as possible for all frequencies to eliminate the amplitude distortion. Fig. 7 illustrates the magnitude responses of $U_{i}(z), i=0,1, \cdots, 2 M-1$. Recalling the assumption that $U_{i}(z)$ has high stopband attenuation and there is no passband or transition band overlap between $U_{i}(z)$ and $U_{i^{\prime}}(z)$ 
when $\left|i-i^{\prime}\right|>1$, only $U_{i}(z)$ and $U_{i+1}(z)$ contribute to $|T(z)|$ in the frequency interval $(i+0.5) \pi / M \leq \omega \leq(i+1.5) \pi / M$. This indicates that if we force $\left|U_{i}(z)\right|^{2}+\left|U_{i+1}(z)\right|^{2}$ to be sufficiently flat in the frequency interval $(i+0.5) \pi / M \leq \omega \leq(i+1.5) \pi / M,|T(z)|$ will be flat enough for all frequencies. Noticing in (7) that $U_{i}(z)$ is a frequency-shifted version of $P(z)$, $\left|U_{i}(z)\right|^{2}+\left|U_{i+1}(z)\right|^{2}$ is also a shifted version of $|P(z)|^{2}+\left|P\left(z W_{2 M}^{1}\right)\right|^{2}$, i.e. $\left|P\left(e^{j \omega}\right)\right|^{2}+\left|P\left(e^{j(\omega-\pi / M)}\right)\right|^{2}$ in the frequency domain, with the same offset. As a result, the proposed SI-NCMFB can achieve an acceptable amplitude by minimizing the following objective function,

$$
\psi=\alpha \int_{0}^{\pi / M}\left(\left|P\left(e^{j \omega}\right)\right|^{2}+\left|P\left(e^{j\left(\omega-\frac{\pi}{M}\right)}\right)\right|^{2}-1\right)^{2} d \omega+(1-\alpha) \int_{\frac{\pi}{2 M}+\varepsilon}^{\pi}\left|P\left(e^{j \omega}\right)\right|^{2} d \omega .
$$

The first term reflects the flatness of $|T(z)|$, and the second term measures the stopband attenuation of $P(z) . \alpha$ is a tradeoff parameter with $0<\alpha<1 . \varepsilon$ is constrained in the interval $[0, \pi / 2 M]$ to ensure that $U_{i}(z)$ and $U_{i^{\prime}}(z)$ do not overlap in the passband or transition band when $\left|i-i^{\prime}\right|>1$. This optimization problem, which involves only one prototype filter, has been widely studied and many efficient methods such as iterative least square approach [24] and convex optimization [25] are developed. In this work, we employ the convex optimization method to solve it.

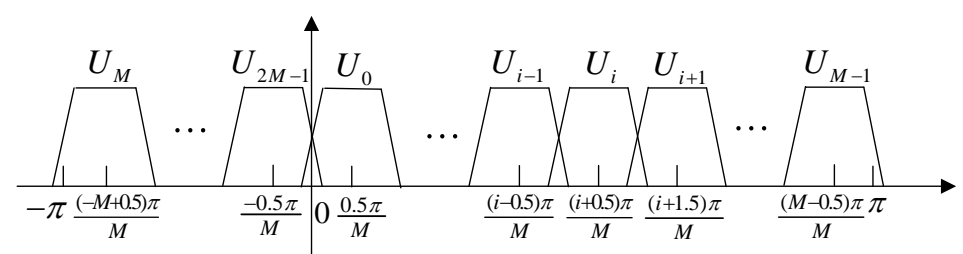

Fig. 7. Magnitude responses of $U_{i}(z), i=0,1, \cdots, 2 M-1$.

\subsection{Efficient implementation}

The proposed SI-NCMFB not only has low design complexity but can also be implemented efficiently by using polyphase implementation structure. In this subsection, we measure the implementation complexity of the SI-NCMFB by the number of multiplications and additions per input sample. Since the synthesis part has the same complexity as the analysis part, we focus only on the analysis part. 
Consider the analysis part of the $k$ th channels of the first and second subsystems in Fig. 8(a). The analysis filters $H_{k}(z)$ and $H_{k}^{\prime}(z)$ have the following Type 1 polyphase representation [26],

$$
\left[\begin{array}{l}
H_{k}(z) \\
H_{k}^{\prime}(z)
\end{array}\right]=\underbrace{\left[\begin{array}{llll}
E_{k, 0}\left(z^{n_{k}}\right) & E_{k, 1}\left(z^{n_{k}}\right) & \cdots & E_{k, n_{k}-1}\left(z^{n_{k}}\right) \\
E_{k, 0}^{\prime}\left(z^{n_{k}}\right) & E_{k, 1}^{\prime}\left(z^{n_{k}}\right) & \cdots & E_{k, n_{k}-1}^{\prime}\left(z^{n_{k}}\right)
\end{array}\right]}_{\mathbf{E}_{k}\left(z^{n_{k}}\right)}\left[\begin{array}{llll}
1 & z^{-1} & \cdots & z^{-\left(n_{k}-1\right)}
\end{array}\right]^{T},
$$

where the superscript $\mathrm{T}$ denotes transpose, and $E_{k, m}(z)=\sum_{n} h_{k}\left(n_{k} n+m\right) z^{-n}$ with $h_{k}(n)$ representing the impulse responses of $H_{k}(z)$. By invoking the Noble identity, Fig. 8(a) is redrawn as in Fig. 8(b). By using such a structure, the number of required multiplications and additions for the analysis part of the $k$ th channels of the two subsystems is $2(N+1) / n_{k}$ and $2 N / n_{k}$, respectively. Therefore, polyphase implementation of the whole analysis part requires a total number of $\sum_{k=0}^{K-1} 2(N+1) / n_{k}=2(N+1)$ multiplications and $\sum_{k=0}^{K-1} 2 N / n_{k}=2 N$ additions. They depend only on the prototype order $N$ and the system redundancy 2 .

The traditional oversampled NUFBs can also be implemented with polyphase structure. In the case of similar filter order and similar system redundancy, the SI-NCMFB has the comparable complexity to the NUFBs designed by the joining method [14-16] and the merging method [20,21], but lower than those designed by the warping method for their involved additional all-pass filters [17-19].

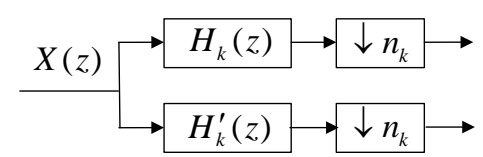

(a)

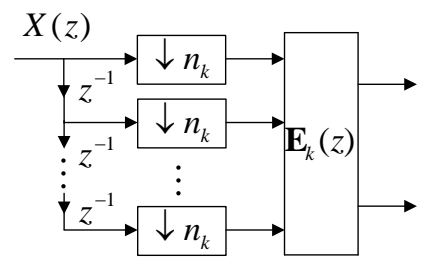

(b)

Fig. 8. Analysis part of the $k$ th channels of the two subsystems. (a) Direct implementation; (b) polyphase implementation.

\section{Directional-selectivity of 2D SI-NCMFB}

As well known, directional-selectivity is highly desired in directional image representations. However, the traditional 2D NUFBs are usually achieved by separable filtering along columns and then along rows. Each resulting subband has the frequency support in all four quadrants of the 2D frequency plane and thus is lack of directional-selectivity. Differently, the proposed SI-NCMFB can 
provide flexible directional-selectivity in two dimensions via separable operations.

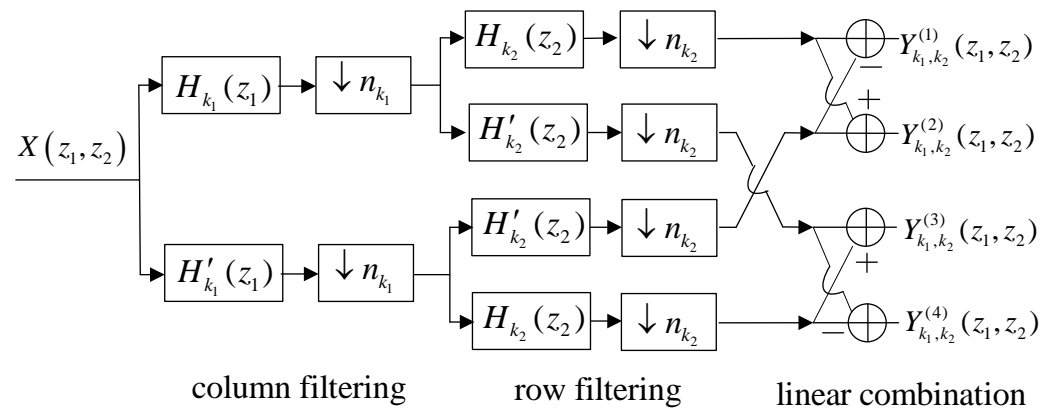

(a)

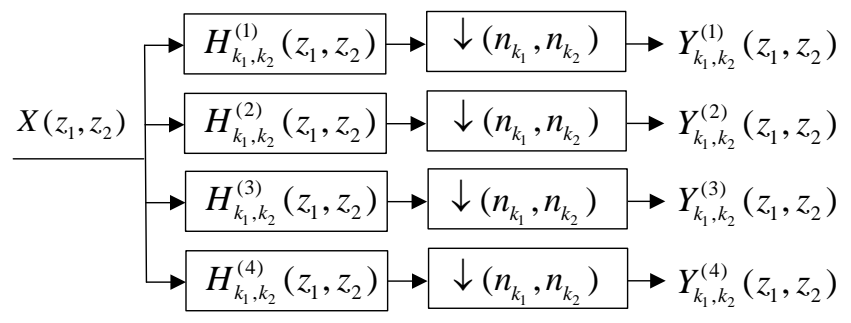

(b)

Fig. 9. (a) Analysis part of 2D SI-NCMFB, (b) its equivalent structure. For a clear illustration, only the $k_{1}$ th channel filtering along columns and the $k_{2}$ th channel filtering along rows are presented, $k_{1}, k_{2}=0,1, \cdots, K-1$.

By separable filtering along columns and rows followed by linear combinations, the SI-NCMFB is extended to two dimensions. Without loss of generality, we show the analysis part of the $k_{1}$ th channel filtering along columns and the $k_{2}$ th channel filtering along rows in Fig. $9(\mathrm{a}), k_{1}, k_{2}=0,1, \cdots, K-1$. Its equivalent structure is illustrated in Fig. 9(b) with the block $\downarrow\left(n_{k_{1}}, n_{k_{2}}\right)$ denoting the separable sampling matrix $\downarrow\left[n_{k_{1}}, 0 ; 0, n_{k_{2}}\right]$ and

$$
\begin{aligned}
& H_{k_{1}, k_{2}}^{(1)}\left(z_{1}, z_{2}\right)=H_{k_{1}}\left(z_{1}\right) H_{k_{2}}\left(z_{2}\right)-H_{k_{1}}^{\prime}\left(z_{1}\right) H_{k_{2}}^{\prime}\left(z_{2}\right), \\
& H_{k_{1}, k_{2}}^{(2)}\left(z_{1}, z_{2}\right)=H_{k_{1}}\left(z_{1}\right) H_{k_{2}}\left(z_{2}\right)+H_{k_{1}}^{\prime}\left(z_{1}\right) H_{k_{2}}^{\prime}\left(z_{2}\right), \\
& H_{k_{1}, k_{2}}^{(3)}\left(z_{1}, z_{2}\right)=H_{k_{1}}^{\prime}\left(z_{1}\right) H_{k_{2}}\left(z_{2}\right)+H_{k_{1}}\left(z_{1}\right) H_{k_{2}}^{\prime}\left(z_{2}\right), \\
& H_{k_{1}, k_{2}}^{(4)}\left(z_{1}, z_{2}\right)=H_{k_{1}}^{\prime}\left(z_{1}\right) H_{k_{2}}\left(z_{2}\right)-H_{k_{1}}\left(z_{1}\right) H_{k_{2}}^{\prime}\left(z_{2}\right) .
\end{aligned}
$$

The linear combination operations in Fig. 9(a) are included in these equivalent analysis filters $H_{k_{1}, k_{2}}^{(1)}\left(z_{1}, z_{2}\right), H_{k_{1}, k_{2}}^{(2)}\left(z_{1}, z_{2}\right), H_{k_{1}, k_{2}}^{(3)}\left(z_{1}, z_{2}\right)$ and $H_{k_{1}, k_{2}}^{(4)}\left(z_{1}, z_{2}\right)$. To analyze the directional-selectivity of the subbands $Y_{k_{1}, k_{2}}^{(1)}\left(z_{1}, z_{2}\right), Y_{k_{1}, k_{2}}^{(2)}\left(z_{1}, z_{2}\right), Y_{k_{1}, k_{2}}^{(3)}\left(z_{1}, z_{2}\right)$ and $Y_{k_{1}, k_{2}}^{(4)}\left(z_{1}, z_{2}\right)$, it is equivalent to analyze the 
directional-selectivity of those filters in (17). For simple analysis, we denote $\sum_{i=l_{k}}^{l_{k}+m_{k}-1} a_{i} U_{i}(z) / \sqrt{m_{k}}$ by $P_{k}(z)$ and $\sum_{i=l_{k}}^{l_{k}+m_{k}-1} a_{i}^{*} U_{i}(z) / \sqrt{m_{k}}$ by $Q_{k}(z)$. Substituting (8) and (9) into (17), we have

$$
\begin{gathered}
H_{k_{1}, k_{2}}^{(1)}\left(z_{1}, z_{2}\right)=2\left(P_{k_{1}}\left(z_{1}\right) P_{k_{2}}\left(z_{2}\right)+P_{k_{1}}^{*}\left(z_{1}\right) P_{k_{2}}^{*}\left(z_{2}\right)\right), \\
H_{k_{1}, k_{2}}^{(2)}\left(z_{1}, z_{2}\right)=2\left(P_{k_{1}}\left(z_{1}\right) P_{k_{2}}^{*}\left(z_{2}\right)+P_{k_{1}}^{*}\left(z_{1}\right) P_{k_{2}}\left(z_{2}\right)\right), \\
H_{k_{1}, k_{2}}^{(3)}\left(z_{1}, z_{2}\right)=-j 2\left(P_{k_{1}}\left(z_{1}\right) P_{k_{2}}\left(z_{2}\right)-P_{k_{1}}^{*}\left(z_{1}\right) P_{k_{2}}^{*}\left(z_{2}\right)\right), \\
H_{k_{1}, k_{2}}^{(4)}\left(z_{1}, z_{2}\right)=-j 2\left(P_{k_{1}}\left(z_{1}\right) P_{k_{2}}^{*}\left(z_{2}\right)-P_{k_{1}}^{*}\left(z_{1}\right) P_{k_{2}}\left(z_{2}\right)\right) .
\end{gathered}
$$

Since $P_{k}(z)$ has the frequency support $\left[\sum_{i=0}^{k-1} \pi / n_{i}, \sum_{i=0}^{k} \pi / n_{i}\right]$, the frequency support of $H_{k_{1}, k_{2}}^{(1)}\left(z_{1}, z_{2}\right)$ and $H_{k_{1}, k_{2}}^{(3)}\left(z_{1}, z_{2}\right)$ is $\left[-\sum_{i=0}^{k_{1}} \pi / n_{i},-\sum_{i=0}^{k_{1}-1} \pi / n_{i}\right] \times\left[-\sum_{i=0}^{k_{2}} \pi / n_{i},-\sum_{i=0}^{k_{2}-1} \pi / n_{i}\right] \cup\left[\sum_{i=0}^{k_{1}-1} \pi / n_{i}, \sum_{i=0}^{k_{1}} \pi / n_{i}\right] \times$ $\left[\sum_{i=0}^{k_{2}-1} \pi / n_{i}, \sum_{i=0}^{k_{2}} \pi / n_{i}\right]$ and that of $H_{k_{1}, k_{2}}^{(2)}\left(z_{1}, z_{2}\right)$ and $H_{k_{1}, k_{2}}^{(4)}\left(z_{1}, z_{2}\right)$ is $\left[-\sum_{i=0}^{k_{1}} \pi / n_{i},-\sum_{i=0}^{k_{1}-1} \pi / n_{i}\right] \times$ $\left[\sum_{i=0}^{k_{2}-1} \pi / n_{i}, \sum_{i=0}^{k_{2}} \pi / n_{i}\right] \cup\left[\sum_{i=0}^{k_{1}-1} \pi / n_{i}, \sum_{i=0}^{k_{1}} \pi / n_{i}\right] \times\left[-\sum_{i=0}^{k_{2}} \pi / n_{i},-\sum_{i=0}^{k_{2}-1} \pi / n_{i}\right]$, which are sketched in Fig. 10. It is obvious that all of them are directionally selective. $H_{k_{1}, k_{2}}^{(1)}\left(z_{1}, z_{2}\right)$ and $H_{k_{1}, k_{2}}^{(3)}\left(z_{1}, z_{2}\right)$ are oriented in the direction of $\theta=\arctan \left[\left(\sum_{i=0}^{k_{2}-1} \pi / n_{i}+\pi / 2 n_{k_{2}}\right) /\left(\sum_{i=0}^{k_{1}-1} \pi / n_{i}+\pi / 2 n_{k_{1}}\right)\right], \quad$ and $H_{k_{1}, k_{2}}^{(2)}\left(z_{1}, z_{2}\right)$ and $H_{k_{1}, k_{2}}^{(4)}\left(z_{1}, z_{2}\right)$ are oriented at $\beta=\pi-\theta$.

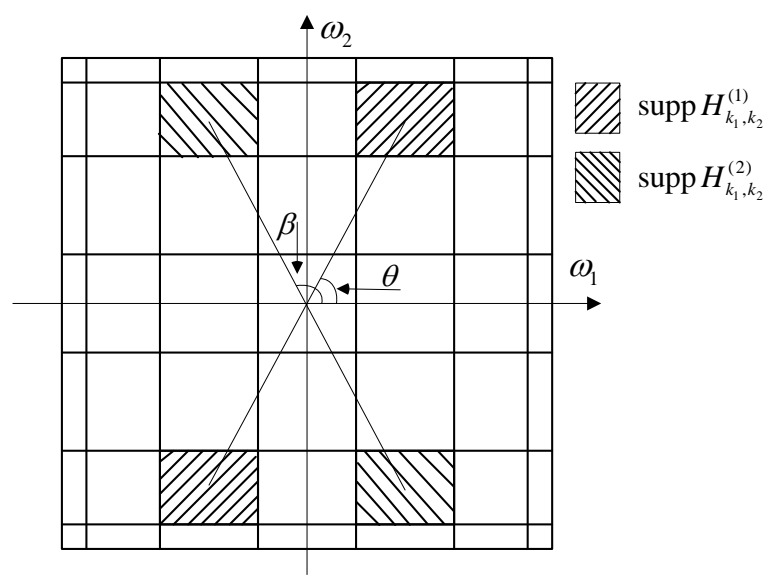

Fig. 10. Illustrations of the frequency supports of $H_{k_{1}, k_{2}}^{(1)}\left(z_{1}, z_{2}\right)$ and $H_{k_{1}, k_{2}}^{(2)}\left(z_{1}, z_{2}\right)$ where $\theta=\arctan \left[\left(\sum_{i=0}^{k_{2}-1} \pi / n_{i}+\pi / 2 n_{k_{2}}\right) /\left(\sum_{i=0}^{k_{1}-1} \pi / n_{i}+\pi / 2 n_{k_{1}}\right)\right]$ and $\beta=\pi-\theta . H_{k_{1}, k_{2}}^{(3)}\left(z_{1}, z_{2}\right)$ and $H_{k_{1}, k_{2}}^{(4)}\left(z_{1}, z_{2}\right)$ have the same frequency supports with $H_{k_{1}, k_{2}}^{(1)}\left(z_{1}, z_{2}\right)$ and $H_{k_{1}, k_{2}}^{(2)}\left(z_{1}, z_{2}\right)$, respectively. 
Due to the fact that the 1D SI-NCMFB has arbitrary integer sampling factors, the resulting 2D SINCMFB can have flexible nonuniform directional frequency partition and thus has the potential to extract the image directional information efficiently. In addition, with the similar analysis on the shiftinvariance of 1D SI-NCMFB, the aliasing terms caused by downsampling the output of $H_{k_{1}, k_{2}}^{(1)}\left(z_{1}, z_{2}\right)$ can be cancelled with those caused by downsampling the output of $H_{k_{1}, k_{2}}^{(3)}\left(z_{1}, z_{2}\right)$, and the aliasing terms caused by downsampling the output of $H_{k_{1}, k_{2}}^{(2)}\left(z_{1}, z_{2}\right)$ can be cancelled with those caused by downsampling the output of $H_{k_{1}, k_{2}}^{(4)}\left(z_{1}, z_{2}\right)$. Therefore, the 2D SI-NCMFB also preserves the shiftinvariance property in one dimension.

\section{Simulation results}

In this section, we firstly take several examples including both the feasible and nonfeasible cases to evaluate the reconstruction performance and shift-invariance of the proposed SI-NCMFB, and then apply 2D SI-NCMFB in directional feature extraction to illustrate its flexible directional-selectivity.

\subsection{Reconstruction performance evaluation}

Feasible partition case: Consider a 5-channel SI-NCMFB with the integer sampling factors $\{9,9,9,3,3\}$. It has the frequency partition $[0, \pi / 9] \cup[\pi / 9,2 \pi / 9] \cup[2 \pi / 9, \pi / 3] \cup[\pi / 3,2 \pi / 3] \cup$ $[2 \pi / 3, \pi]$ and thus satisfies the feasible frequency partition constraint as shown in Fig. 4 . The lcm of $\{9,9,9,3,3\}$ is $M=9$. A linear-phase prototype filter $P(z)$ with the frequency support $[-\pi / 18, \pi / 18]$ and the order $N=53$ is firstly designed. Its stopband edge is set as $\omega_{s}=0.11 \pi$ to ensure that there is no passband or transition band overlap between the shifted versions of the prototype filter. Fig. 11(a) shows the prototype magnitude response with the stopband attenuation $33 \mathrm{~dB}$. All the involved filters of the first and second subsystems are constructed from (8) and (9). Figs. 11(b) and (c) show the magnitude responses of $H_{k}(z)$ and $H_{k}^{\prime}(z)$, respectively, $k=0,1, \cdots, 4$. As for the reconstruction performance of the whole system, we employ the maximum peak-to-peak values of the amplitude distortion $|T(z)|-1$, denoted by $E_{p p}$, and the maximum value of the total aliasing error 
$\sqrt{\sum_{k=0}^{K-1} \sum_{l=1}^{n_{k}-1}\left|A_{k, l}(z)\right|}$, denoted by $E_{a}$, to evaluate it. They measure the worst amplitude distortion and the worst peak aliasing error, respectively. Fig. 11(d) plots the amplitude distortion $|T(z)|-1$ with $E_{p p}=1.36 \times 10^{-2}$ and Fig. 11(e) displays the total aliasing error $\sqrt{\sum_{k=0}^{K-1} \sum_{l=1}^{n_{k}-1}\left|A_{k, l}(z)\right|}$ with $E_{a}=6.88 \times 10^{-4}$.

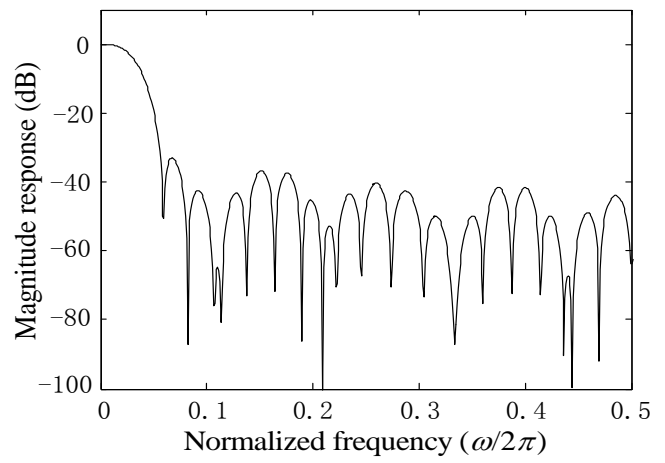

(a)

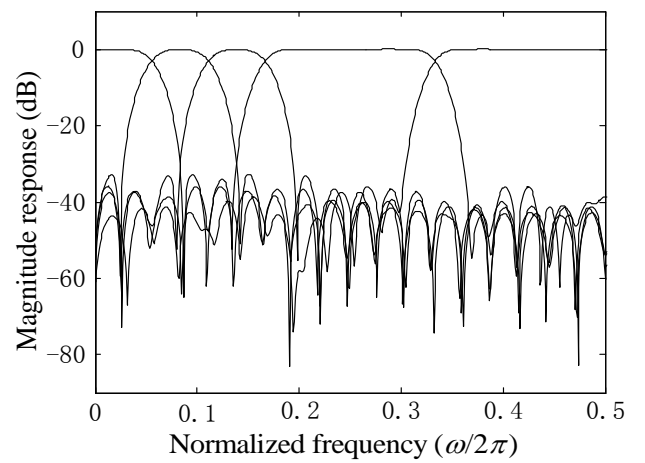

(b)

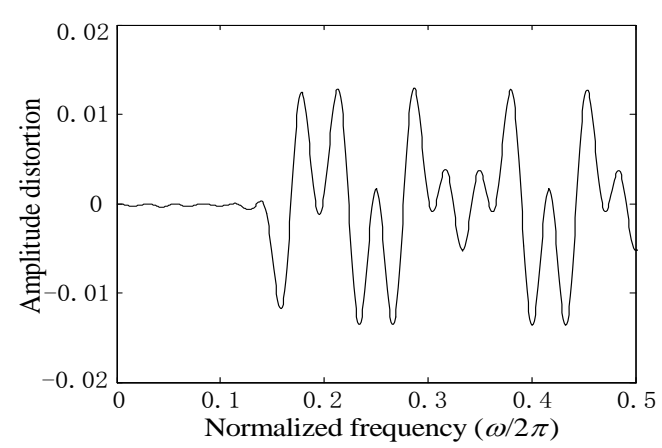

(d)

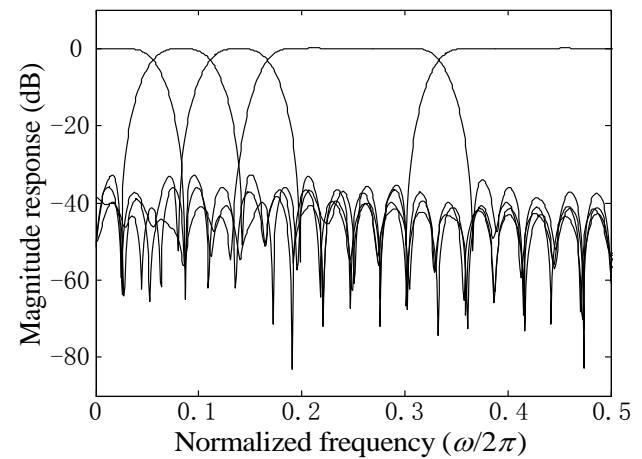

(c)

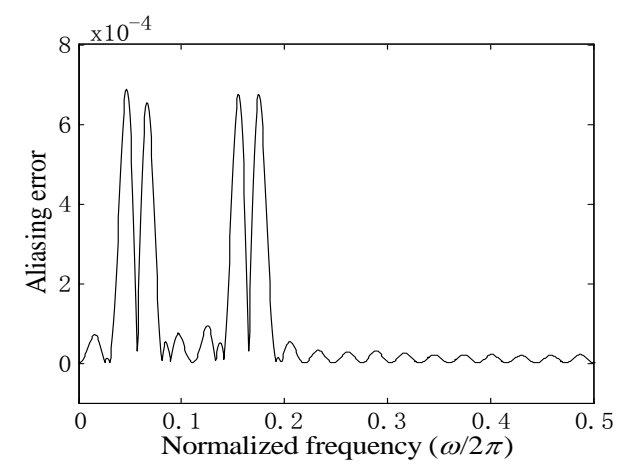

(e)

Fig. 11. SI-NCMFB with integer sampling factors $\{9,9,9,3,3\}$. (a) Magnitude response of prototype filter, (b) magnitude responses of analysis filters of the first system, (c) magnitude responses of analysis filters of the second system, (d) amplitude distortion, (f) aliasing error.

Nonfeasible partition case: Consider a 5-channel SI-NCMFB with the integer sampling factors 


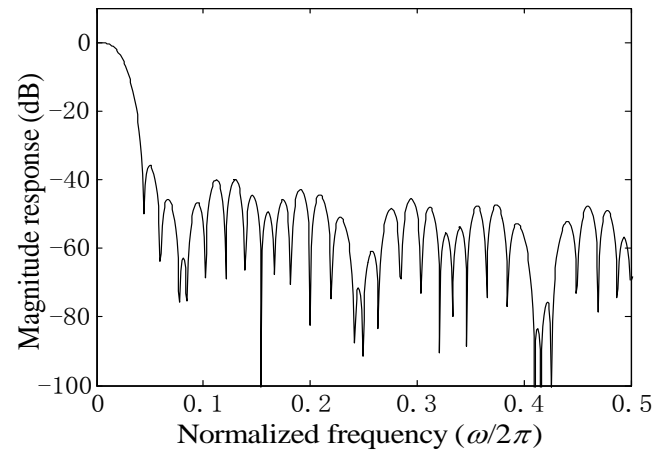

(a)

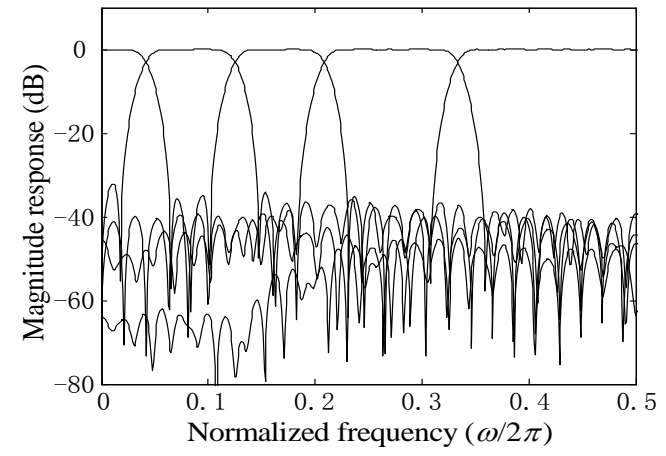

(b)

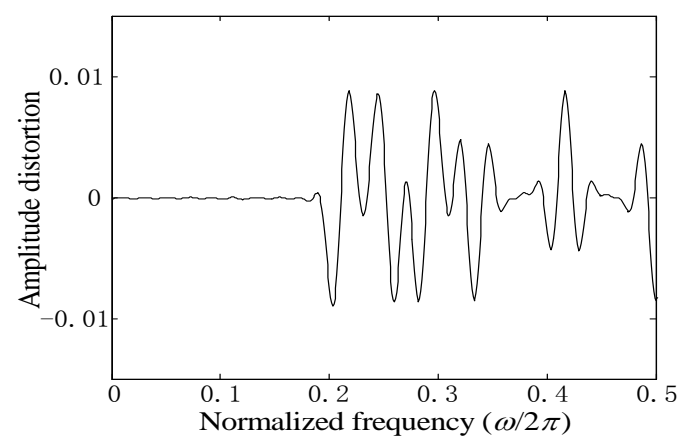

(d)

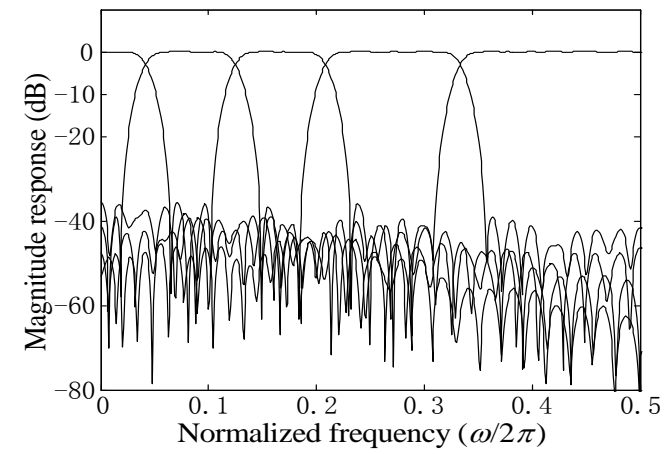

(c)

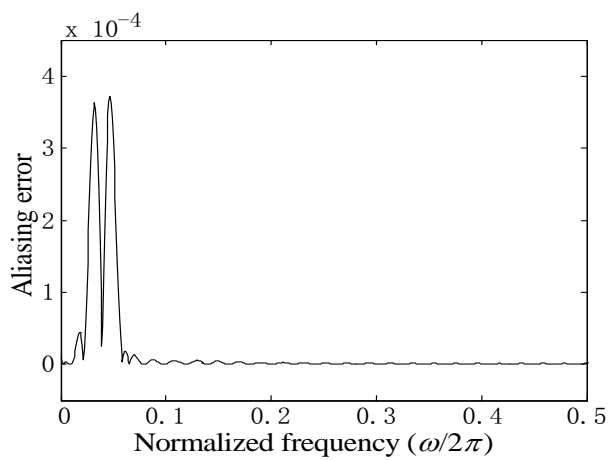

(e)

Fig. 12. SI-NCMFB with sampling factors $\{12,6,6,4,3\}$. (a) Magnitude response of prototype filter, (b) magnitude responses of analysis filters of the first subsystem, (c) magnitude responses of analysis filters of the second subsystem, (d) amplitude distortion, (f) aliasing error.

$\{12,6,6,4,3\}$ and the frequency partition $[0, \pi / 12] \cup[\pi / 12, \pi / 4] \cup[\pi / 4,5 \pi / 12] \cup[5 \pi / 12,2 \pi / 3] \cup$ $[2 \pi / 3, \pi]$. It can be verified that the $k$ th channel violates the feasible frequency partition constraint, $k=1,2,3$. Since the lcm of $\{12,6,6,4,3\}$ is $M=12$, a linear-phase prototype filter $P(z)$ with the frequency support $[-\pi / 24, \pi / 24]$ is firstly designed (see Fig. 12(a)). It has the order $N=71$, the stopband edge $\omega_{s}=0.08 \pi$, and the stopband attenuation $36 \mathrm{~dB}$. According to (8) and (9), all the analysis and synthesis filters are obtained as shown in Figs. 12(b) and (c). Figs. 12(d) and (e) show the 
amplitude distortion and the aliasing error of the whole system with $E_{p p}=8.96 \times 10^{-3}$ and $E_{a}=3.71 \times 10^{-4}$.

From the amplitude distortion and aliasing error of the above two examples, it can be seen that the SI-NCMFBs with both feasible and nonfeasible frequency partitions are of NPR. Such distortions are acceptable in various signal and image applications such as noise reduction and feature extraction.

\subsection{Shift-invariance evaluation}

This subsection takes the two SI-NCMFBs just designed in Section 5.1 to evaluate the performance of shift-invariance. One SI-NCMFB has feasible frequency partition and the other has nonfeasible frequency partition. For a filter bank, the shift-invariance of each channel is usually tested via the ratio of the total energy of unwanted aliasing transfer functions to the energy of wanted transfer function [22]. Since the energy of a signal can be calculated in the frequency domain from Parseval's theorem, we formulate the energy ratio of the $k$ th channels of the two subsystems as

$$
R_{k}=\frac{\sum_{l=1}^{n_{k}-1} \varepsilon\left\{H_{k}\left(z W_{n_{k}}^{l}\right) F_{k}(z)+H_{k}^{\prime}\left(z W_{n_{k}}^{l}\right) F_{k}^{\prime}(z)\right\}}{\varepsilon\left\{H_{k}(z) F_{k}(z)+H_{k}^{\prime}(z) F_{k}^{\prime}(z)\right\}},
$$

where $\varepsilon(B(z))$ calculates the energy of the impulse response of $B(z)$ in the frequency domain with $\varepsilon(B(z))=1 / 2 \pi \cdot \int_{-\pi}^{\pi}\left|B\left(e^{j \omega}\right)\right|^{2} d \omega$. The smaller $R_{k}$ is, the less aliasing remains and the better shiftinvariance performance is.

\section{Table 1}

Energy ratios $R_{k}$ in dB for two SI-NCMFBs with sampling factors $\{9,9,9,3,3\}$ and $\{12,6,6,4,3\}$.

\begin{tabular}{c|ccccc}
\hline \multirow{2}{*}{ SI-NCMFB } & \multicolumn{5}{|c}{ Channel index } \\
\cline { 2 - 6 } & $k=0$ & $k=1$ & $k=2$ & $k=3$ & -39.32 \\
\hline Feasible case $\{9,9,9,3,3\}$ & -39.42 & -35.42 & -35.19 & -39.41 & -48.61 \\
Nonfeasible case $\{12,6,6,4,3\}$ & -40.70 & -35.38 & -35.37 & -41.52 & -40 \\
\hline \hline
\end{tabular}

Tab. 1 tabulates the energy ratios of the two SI-NCMFBs. For convenience, we represent $R_{k}$ in $\mathrm{dB}$. From Tab. 1 it can be seen that, the energy ratios $R_{k}$ are about $-41 \sim-35 \mathrm{~dB}$, that is to say, the energy of all the aliasing transfer functions is only about $0.008 \% \sim 0.03 \%$ of the energy of the wanted transfer function. It means that the aliasing in the first and second subsystems is cancelled by each other satisfactorily, and the two SI-NCMFBs with both feasible and nonfeasible frequency partitions have 
good shift-invariance performance.

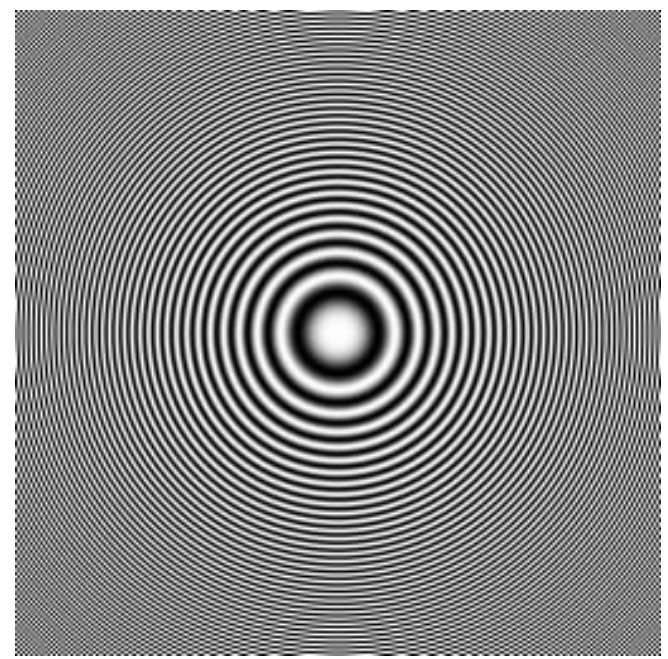

(a)

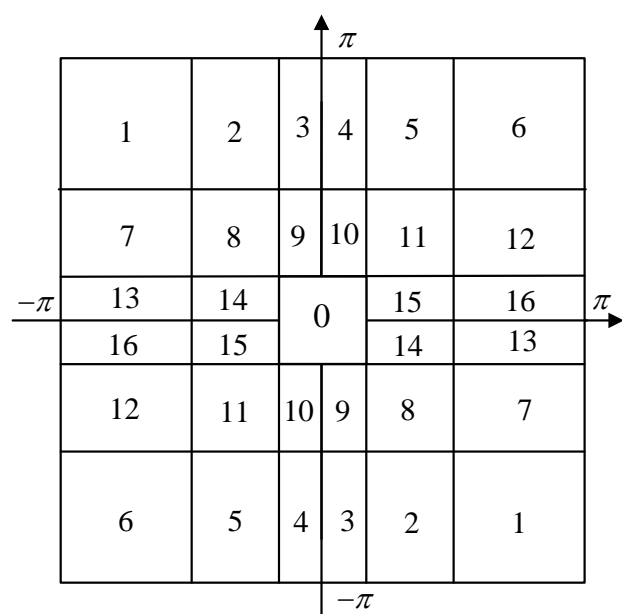

(b)

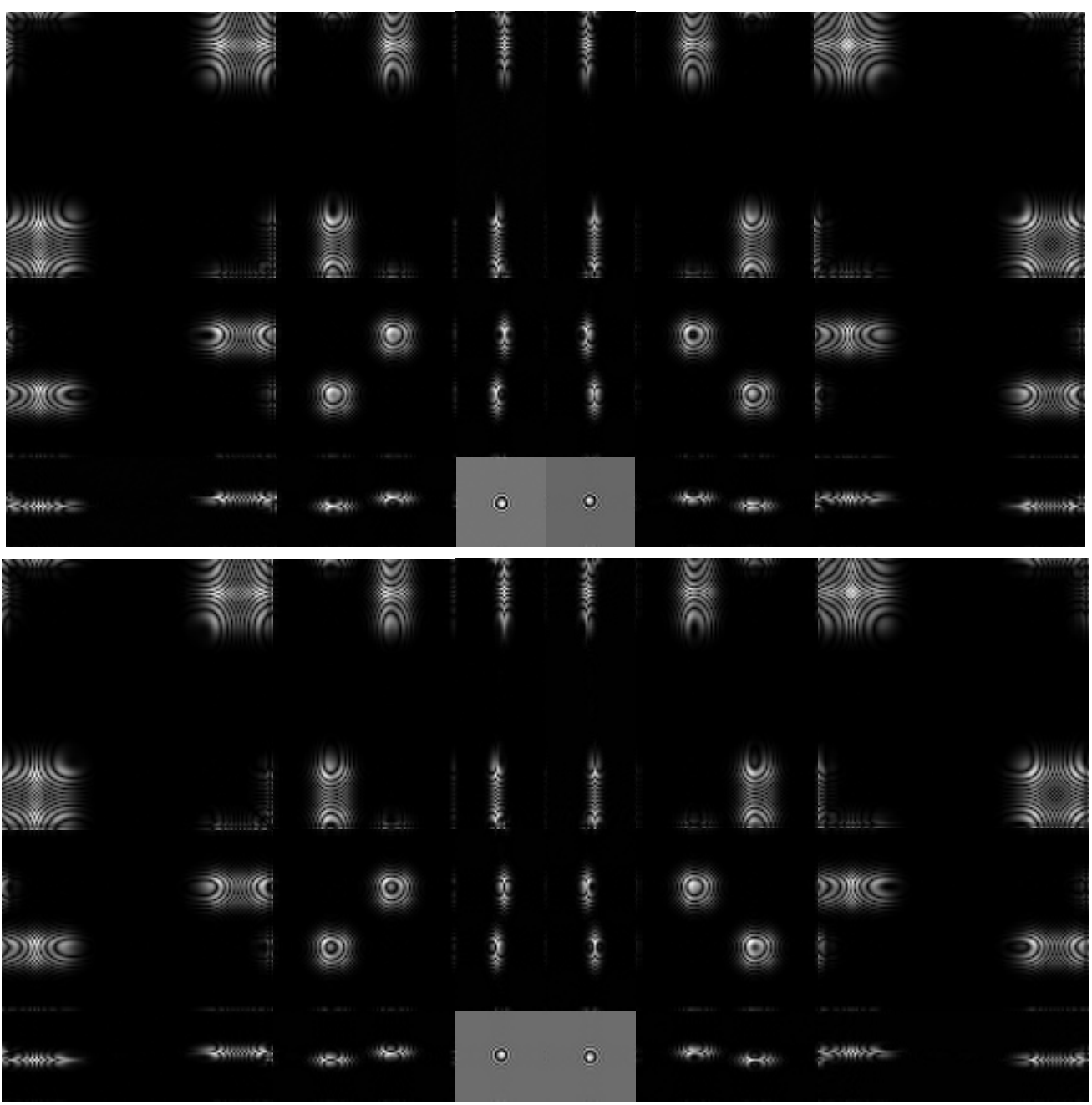

(c)

Fig. 13. Directional feature extraction. (a) Test image Zoneplate, (b) frequency partition of the 2D SI-NCMFB with sampling factors $\{6,3,2\}$, (c) the resulting subbands, where the upper sixteen directional subbands come from $Y_{k_{1}, k_{2}}^{(1)}\left(z_{1}, z_{2}\right)$ and $Y_{k_{1}, k_{2}}^{(2)}\left(z_{1}, z_{2}\right)$ and the lower directional ones are from $Y_{k_{1}, k_{2}}^{(3)}\left(z_{1}, z_{2}\right)$ and $Y_{k_{1}, k_{2}}^{(4)}\left(z_{1}, z_{2}\right)$. 


\subsection{Directional feature extraction}

To illustrate the flexible directional-selectivity of 2D SI-NCMFB, a 3-channel SI-NCMFB with the integer sampling factors $\{6,3,2\}$ is employed to decompose the test image Zoneplate (see Fig. 13(a)) which has the frequency content from dc to $\pm \pi$. By separable filtering along columns and rows followed by linear combinations, we extend this SI-NCMFB from one dimension to two dimensions. Fig. 13(b) shows the frequency partition scheme of the 2D SI-NCMFB. As a result, the test image is decomposed into four lowpass subbands and thirty directional subbands. Fig. 13(c) displays all the subband coefficients, where the upper sixteen directional subbands come from $Y_{k_{1}, k_{2}}^{(1)}\left(z_{1}, z_{2}\right)$ and $Y_{k_{1}, k_{2}}^{(2)}\left(z_{1}, z_{2}\right)$ and the lower ones are from $Y_{k_{1}, k_{2}}^{(3)}\left(z_{1}, z_{2}\right)$ and $Y_{k_{1}, k_{2}}^{(4)}\left(z_{1}, z_{2}\right)$.

This example illustrates that the 2D SI-NCMFB can provide flexible directional-selectivity and thus has the potential to efficiently represent images with abundant directional features. This cannot be performed by conventional 2D oversampled NUFBs.

\section{Conclusions}

This paper presents a simple but efficient design method for SI-NCMFB. The significant aliasing appearing in the first and second subsystems approximately cancel each other, resulting in the shiftinvariance property and arbitrary integer sampling factors. Moreover, both the first and second subsystems are derived from just one linear-phase prototype filter, reducing the design complexity significantly. By extending the SI-NCMFB to two dimensions via separable operations, the 2D SINCMFB provides flexibly directional-selectivity. Several experiments verify that the SI-NCMFB is a NPR system, and has good shift-invariance property and flexibly nonuniform frequency partition. The experiment on directional feature extraction indicates its potential in representation of images which contain abundant directional features.

\section{Appendix A}

In this appendix, we give the derivation of $U_{i}(z)=z^{-N} U_{i}^{*}\left(z^{-1}\right)$. According to the expression of $U_{i}(z)$ in (7), $z^{-N} U_{i}^{*}\left(z^{-1}\right)$ can be expressed as 


$$
z^{-N} U_{i}^{*}\left(z^{-1}\right)=z^{-N} W_{2 M}^{-(i+0.5) N / 2} P\left(z^{-1} W_{2 M}^{-(i+0.5)}\right)
$$

Since $P(z)$ is a lowpass linear-phase prototype filter with the order of $N$, its impulse responses are symmetric, or equivalently in the $Z$ domain it satisfies

$$
P(z)=z^{-N} P\left(z^{-1}\right)
$$

Replacing $z$ with $z W_{2 M}^{i+0.5}$ in $(21)$, we have

$$
P\left(z W_{2 M}^{i+0.5}\right)=z^{-N} W_{2 M}^{-(i+0.5) N} P\left(z^{-1} W_{2 M}^{-(i+0.5)}\right)
$$

Multiplying both sides of (22) by $W_{2 M}^{(i+0.5) N / 2}$, Eq. (22) is changed as

$$
W_{2 M}^{(i+0.5) N / 2} P\left(z W_{2 M}^{i+0.5}\right)=z^{-N} W_{2 M}^{-(i+0.5) N / 2} P\left(z^{-1} W_{2 M}^{-(i+0.5)}\right) .
$$

Observing (7) and (20), it can be seen that the left side of (23) is just $U_{i}(z)$, and the right side is just $z^{-N} U_{i}^{*}(z)$. Finally we derive that

$$
U_{i}(z)=z^{-N} U_{i}^{*}\left(z^{-1}\right)
$$

\section{References}

[1] J. D. Griesbach, M. Lightner, D. M. Etter, "Subband adaptive filtering decimation constraints for oversampled nonuniform filter banks," IEEE Trans. on Circuits and systems-II: analog and digital signal processing, vol. 49, no. 10, pp. 677-681, Oct. 2002.

[2] S. Cheng, Z. Xiong, "Audio coding and image denoising based on the nonuniform modulated complex lapped transform,” IEEE Trans. on Multimedia, vol. 7, no. 5, pp.817-827, Oct. 2005.

[3] S. J. Darak, J. Palicot, H. Zhang, V. A. Prasad, and C. Moy, "Reconfigurable filter bank with complete control over subband bandwidths for multistandard wireless communication receivers," IEEE Trans. on Very Large Scale Integration Systems, vol. PP, no. 99, pp. 1-11, Sept. 2014.

[4] A. Schasse, T. Gerkmann, R. Martin, W. Sorgel, T. Pilgrim, and H. Puder, "Two-stage filter bank system for improved signal-channel noise reduction in hearing aids," IEEE Trans. on Audio, Speech, and Language processing, vol. 23, no. 2, pp. 383-393, Feb. 2015.

[5] J. Kovacevic, and M. Vetterli, "Perfect reconstruction filter banks with rational sampling factors," IEEE Trans. on Signal Processing, vol. 41, no. 6, pp. 2047-2066, Jun. 1993.

[6] K. Nayebi, T. P. Barnwell, and M. J. T. Smith, "Nonuniform filter banks: a reconstruction and design theory,” IEEE Trans. on Signal Processing, vol. 41, no. 3, pp. 1114-1127, Mar. 1993.

[7] J. Li, T. Q. Nguyen, and S. Tantaratana, "A simple design method for near-perfect-reconstruction nonuniform filter banks,” IEEE Trans. on Signal Processing, vol. 45, no. 8, pp. 2105-2109, Aug. 1997.

[8] X. M. Xie, S. C. Chan, and T. I. Yuk, "Design of perfect-reconstruction nonuniform recombination filter banks with flexible rational sampling factors," IEEE Trans. on Circuits and Systems, vol. 52, No. 9, pp. 1965-1981, Sept. 2005. 
[9] A. Eghbali, H. Johansson, and P. Lowenborg, "Reconfigurable nonuniform transmultiplexers using uniform modulated filter banks," IEEE Trans. on Circuits and Systems, vol. 58, no. 3, pp. 539-547, Mar. 2011.

[10] A. Kumara, G. K. Singhb, and S. Anuraga, "Design of nearly perfect reconstructed non-uniform filter bank by constrained equiripple FIR technique,” Applied Soft Computing, vol. 13, pp. 353-360, 2013.

[11] B. W. Ling, C. Y. Ho, K. Teo, W. Siu, J. Cao, and Q. Dai, "Optimal design of cosine modulated nonuniform linear phase FIR filter bank via both stretching and shifting frequency response of single prototype filter,” IEEE Trans. on Signal Processing, vol. 62, no. 10, pp. 2517-2530, May 2014.

[12] A. Spanias, T. Painter, and V. Atti, Audio Signal Processing and Coding, John Wiley \& Sons, 2007.

[13] M. F. Mansour, “A design procedure for oversampled nonuniform filter banks with perfectreconstruction," In Proc. of IEEE International Conference on Acoustics, Speech and Signal Processing, May 2011, pp. 1649-1652.

[14] Z. Cvetkovic, "Nonuniform oversampled filter banks for audio signal processing," IEEE Trans. on Speech and Audio Processing, vol. 11, no. 5, pp. 393-399, Sept. 2003.

[15] P. Faus, A. Gonzalez, P. Zuccarello, and A. Vidal, "Oversampled nonuniform filter banks using quadratic optimization and transition filters,” Electronics Letters, vol. 43, no. 10, pp. 594-595, May 2007.

[16] B. Dumitrescu, R. Bregovic, and T. Saramaki, "Design of low-delay nonuniform oversampled filterbanks," Signal Processing, vol. 88, pp. 2518-2525, May 2008.

[17] E. Galijasevic and J. Kliewer, "Design of allpass-based nonuniform oversampled DFT filter banks," In Proc. of IEEE International Conference on Acoustics, Speech and Signal Processing, vol. 2, May 2002, pp. 1181-1184.

[18] R. C. Nongpiur and D. J. Shpak, "Maximizing the signal-to-alias ratio in non-uniform filter banks for acoustic echo cancellation,” IEEE Trans. on Circuits and Systems, vol. 59, no. 10, pp. 2315-2325, Oct. 2012.

[19] M. Parfieniuk, and A. Petrovsky, "Near-perfect reconstruction oversampled nonuniform cosine-modulated filter banks based on frequency warping and subband merging," International Journal of Electronics and Telecommunications, vol. 58, no. 2, pp. 177-192, July 2012.

[20] Y. Deng, V. J. Mathews, and B. Farhang-Borouieny, "Low-delay nonuniform pseudo-QMF banks with application to speech enhancement," IEEE Trans. on Signal Processing, vol. 55, no. 5, pp. 2110-2121, May 2007.

[21] S. Kalathil, and E. Elias, "Efficient design of non-uniform cosine modulated filter banks for digital hearing aids," International Journal of Electronics and Communications, vol. 69, pp. 1314-1320, 2015.

[22] N. Kingsbury, "Complex wavelets for shift invariant analysis and filtering of signals," Applied and Computational Harmonic analysis, vol. 10, no. 3, pp. 234-253, May 2001.

[23] T. Q. Nguyen, "Near-perfect -reconstruction pseudo-QMF banks," IEEE Trans. on Signal Processing, vol. 42, no. 1, pp. 65-76, Jan. 1994.

[24] Y. T. Fong, C. W. Kok, "Iterative least squares design of DC-leakage free paraunitary cosine modulated filter banks," IEEE Trans. on Circuits and Systems II: Analog and Digital Signal Processing, vol. 50, no. 5, pp. 238-243, May 2003.

[25] H. H. Kha, H. D. Tuan, and T. Q. Nguyen, "Efficient design of cosine-modulated filter banks via convex optimization,” IEEE Trans. on Signal Processing, vol. 57, no. 3, pp. 966-976, Mar. 2009.

[26] P. P. Vaidyanathan, Multirate Systems and Filter Banks: Englewood Cliffs, NJ: Prentice-Hall, 1993. 